\title{
GROWTH KINETICS OF MYCOBACTERIUM TUBERCULOSIS MEASURED BY QUANTITATIVE RESAZURIN REDUCTION ASSAY: A TOOL FOR FITNESS STUDIES
}

\author{
Andrea von Groll ${ }^{1}$, Anandi Martin ${ }^{1}$, Françoise Portaels ${ }^{1}$, Pedro Eduardo Almeida da Silva ${ }^{2}$, Juan Carlos Palomino ${ }^{1}$ \\ ${ }^{1}$ Mycobacteriology Unit, Institute of Tropical Medicine Antwerp, Nationalestraat 155, B-2000, Antwerp, Belgium; \\ ${ }^{2}$ Laboratorio de Micobacteriologia, Universidade Federal do Rio Grande, Rio Grande, RS, Brasil.
}

Submitted: March 05, 2009; Returned to authors for corrections: May 29, 2009; Approved: November 07, 2009.

\begin{abstract}
We standardized a method to evaluate the growth kinetics of Mycobacterium tuberculosis by measuring quantitatively the reduction of resazurin by spectrophotometry. Growth curves and the rate of growth of twenty-one $M$. tuberculosis clinical isolates were determined. The method showed technical simplicity and is inexpensive to assess the fitness of each isolate.
\end{abstract}

Key words: Growth kinetics, Mycobacterium tuberculosis, Resazurin, Fitness

Determination of growth kinetics of Mycobacterium tuberculosis has wide applications in tuberculosis research. It has been useful in assessing and comparing the fitness of the bacteria in cases of: environmental stress (16), alterations in gene regulation (15) and the presence of gene mutations associated with drug resistance $(5,14)$.

The fact that M. tuberculosis has a slow growth rate and the tendency to form clumps when grown in liquid media, makes it more difficult to study mycobacterial growth by methods commonly used for other bacteria (4). The standard plate count method of viable cells is laborious, time consuming and requires at least 3 weeks to give results and frequently fails due to either contamination or dehydratation of the medium during the long incubation period (3). Turbidity measurement has limited value and works best for well-dispersed cultures containing detergents (7). On the other hand, methods such as the BACTEC radiometric system and the mycobacteria growth indicator tube (MGIT $960^{\mathrm{TM}}$ ) are faster and sensitive $(2,8)$ but require expensive equipment and they are inflexible since they depend on a single pre-packaged growth media $(11,17)$.
In the present study we set up a method to determine the growth kinetic of $M$. tuberculosis by measuring quantitatively the reduction of resazurin by spectrophotometry. Resazurin is a redox indicator dye that is reduced to resorufin by metabolically active cells (1). There is a direct correlation between the reduction of resazurin in the growth medium and the quantity/proliferation of live organisms (9). In $M$. tuberculosis, resazurin has been used successfully to test the susceptibility of active and dormant bacilli to anti-tuberculosis drugs by the resazurin microtiter assay (REMA) plate $(6,10$, 13). Reduction of resazurin can be measured using a spectrophotometer due to its change in colour from blue (oxidized form) to pink (reduced form) (18).

In the present study we propose the use of the REMA format by measuring the optical density (OD) daily with a plate reader. Growth curves were constructed based on the daily differences in the OD values obtained.

In order to standardize the method we compared the growth curve obtained by REMA (REMA-GC) method with the MGIT $960^{\mathrm{TM}}$ system. Five strains, including the reference

*Corresponding Author. Mailing address: Mycobacteriology Unit - Institute of Tropical Medicine Antwerp - Nationalestraat 155 , B-2000 - Antwerp Belgium.; E-mail: avongroll@itg.be 
strain H37Rv, of the Institute of Tropical Medicine strain collection were freshly sub-cultured on Löwenstein-Jensen medium and kept for 3 weeks. An inoculum was prepared at a turbidity of a McFarland tube No.0.5 in ultra pure water and further diluted 1:10 in Middlebrook 7H9 broth supplemented with $0.1 \%$ casitone, $0.5 \%$ glycerol, and $10 \%$ OADC (oleic acid, albumin, dextrose and catalase) (Becton-Dickinson, USA). Resazurin sodium salt powder (Acros Organic NV, Belgium) was prepared at $0.02 \%(\mathrm{wt} / \mathrm{vol})$ in distilled water, sterilized by filtration and stored at $4{ }^{\circ} \mathrm{C}$ in the dark. The REMA method was performed in a sterile flat-bottom 96-well plate to which was added: $200 \mu \mathrm{L}$ of sterile distilled water in all peripheral wells, $200 \mu \mathrm{L}$ of $7 \mathrm{H} 9$ medium containing $10 \%$ OADC in three wells (negative control) and $100 \mu \mathrm{L}$ of $7 \mathrm{H} 9$ with $\mathrm{OADC}+100 \mu \mathrm{L}$ of the inoculum of each strain in another three wells. The plate was closed with its lid, put in sealed plastic bags and incubated at $37^{\circ} \mathrm{C}$. After 48 hours, $30 \mu \mathrm{L}$ of $0.02 \%$ resazurin was added to the wells. The plate was reincubated at $37^{\circ} \mathrm{C}$ and every 24 hours the OD of each well was measured using a plate reader (Biotrak II Visible Plate) at a wavelength of $620 \mathrm{~nm}$. Resazurin is blue and the OD decreases according to the change in colour to pink due to the bacterial metabolism. In order to establish the growth curves, the difference in OD between the average of three wells of the inoculated and the control (uninoculated wells) was plotted versus the time of incubation. The fitness of each strain was estimated by two parameters obtained from the growth curves: the length of the lag phase and the rate of growth. The length of lag phase was determined from the time of starting the incubation until reaching an OD of 0.2 and the rate of growth was the time needed by each strain to reach an OD of 0.4 starting at an OD of 0.2. This time was calculated from the growth curve considering that all strains were in the logarithmic phase of growth between the two OD values.

The determination of growth curve using the MGIT960 ${ }^{\mathrm{TM}}$ was adapted from a method previously reported (14). One hundred $\mu \mathrm{l}$ of the same inoculum used in the REMA-GC method was added, in triplicate, into BBL MGIT ${ }^{\mathrm{TM}}$ Mycobacterial Growth Indicator Tubes supplemented with 10
\% MGIT960 SIRETM Supplement (BD, USA). The tubes were maintained at $37{ }^{\circ} \mathrm{C}$ in the MGIT960 ${ }^{\mathrm{TM}}$ automated system. Growth curves were obtained by monitoring and recording the growth units (GU) every hour using the BD EpiCenter ${ }^{\mathrm{TM}}$ software. The length of lag phase was determined from the time of starting the incubation until reaching a reading of 10 $\mathrm{GU}$ and the rate of growth was determined by the time in hours for a strain to increase from 5,000 to $10,000 \mathrm{GU}$ in the MGIT960'TM system.

Table 1 shows the results of the length of lag phase and rate of growth obtained by the two methods MGIT960 ${ }^{\mathrm{TM}}$ and REMA-GC. The average of the length of lag phase was $133.4 \mathrm{~h}$ and $87.2 \mathrm{~h}$ for MGIT960 ${ }^{\mathrm{TM}}$ and REMA-GC respectively. A shorter length time measured by REMA-GC in comparison to MGIT960"M is probably be due to the higher concentration of the inoculum, since the same inoculum was added in a final volume of $230 \mu \mathrm{L}$ in a well used in the REMA-GC and 7900 $\mu \mathrm{L}$ in a tube used in MGIT960 ${ }^{\mathrm{TM}}$. In both methods, the strain 03-265 presented the longest lag phase and it could be differentiated from the other strains. In case of the rate of growth, the strain $03-850$ presented the fastest rate in both methods; however, it was only significantly faster then the other strains by REMA-GC method. The strain 01-2522 presented the major difference between the two methods. It had the fastest rate by MGIT960 ${ }^{\mathrm{TM}}$ and the slowest by REMA-GC, but the rate of growth remained within the confidence interval for both methods.

A new study with $21 \mathrm{M}$. tuberculosis clinical isolates were performed by REMA-GC method. These strains originated from the Laboratory of Mycobacteria at the Universidade Federal do Rio Grande in Rio Grande, Brazil. The profile of resistance to the first-line drugs (rifampicin, isoniazid, ethambutol and streptomycin) was determined by nitrate reductase assay (12). One strain (06-235) was found multidrugresistant (MDR), that is, resistant to rifampicin and isoniazid. Growth curves of the isolates were obtained through the methodology described above, however the plate reader used was TECAN Spectrum Classic. Figure 1 shows the values of the rate of growth for all 21 clinical isolates. The MDR isolate 
(06-235) presented the slowest rate of growth. Considering that the MDR isolate presented a lower reproductive efficiency; thus, it would be possible to assume that it could be less widely transmitted than the drug susceptible strains. This finding seems to correspond with the situation found in the city of Rio
Grande, where the isolate 06-235 is the only MDR found among 181 clinical isolates obtained from 2006 to 2009 in the national TB control program (data not shown). However, the continuous monitoring of the susceptibility profile of new clinical isolates will allow confirming our finding.

Table 1. Values of the length of lag phase and rate of growth obtained by MGIT960"M and REMA plate methods

\begin{tabular}{ccccc}
\hline & \multicolumn{2}{c}{ Length of lag phase (h) } & \multicolumn{2}{c}{ Rate of growth (h) } \\
\cline { 2 - 5 } & MGIT960 $^{\text {TM }}$ & REMA & MGIT960 $^{\text {TM }}$ & REMA \\
\hline H37Rv & 125 & 68 & 19 & 20 \\
$01-2522$ & 123 & 75 & 15 & 27 \\
$02-2761$ & 125 & 73 & 20 & $17^{*}$ \\
$03-850$ & 131 & 65 & 15 & 24 \\
$03-265$ & $163^{*}$ & $155^{*}$ & 21 & 22.6 \\
Average & 133.4 & 87.2 & 18.0 & $18-27$ \\
99\% CI & $114-153$ & $43-131$ & $15-21$ & \\
\hline
\end{tabular}

*Strains with value out of the confidence interval.

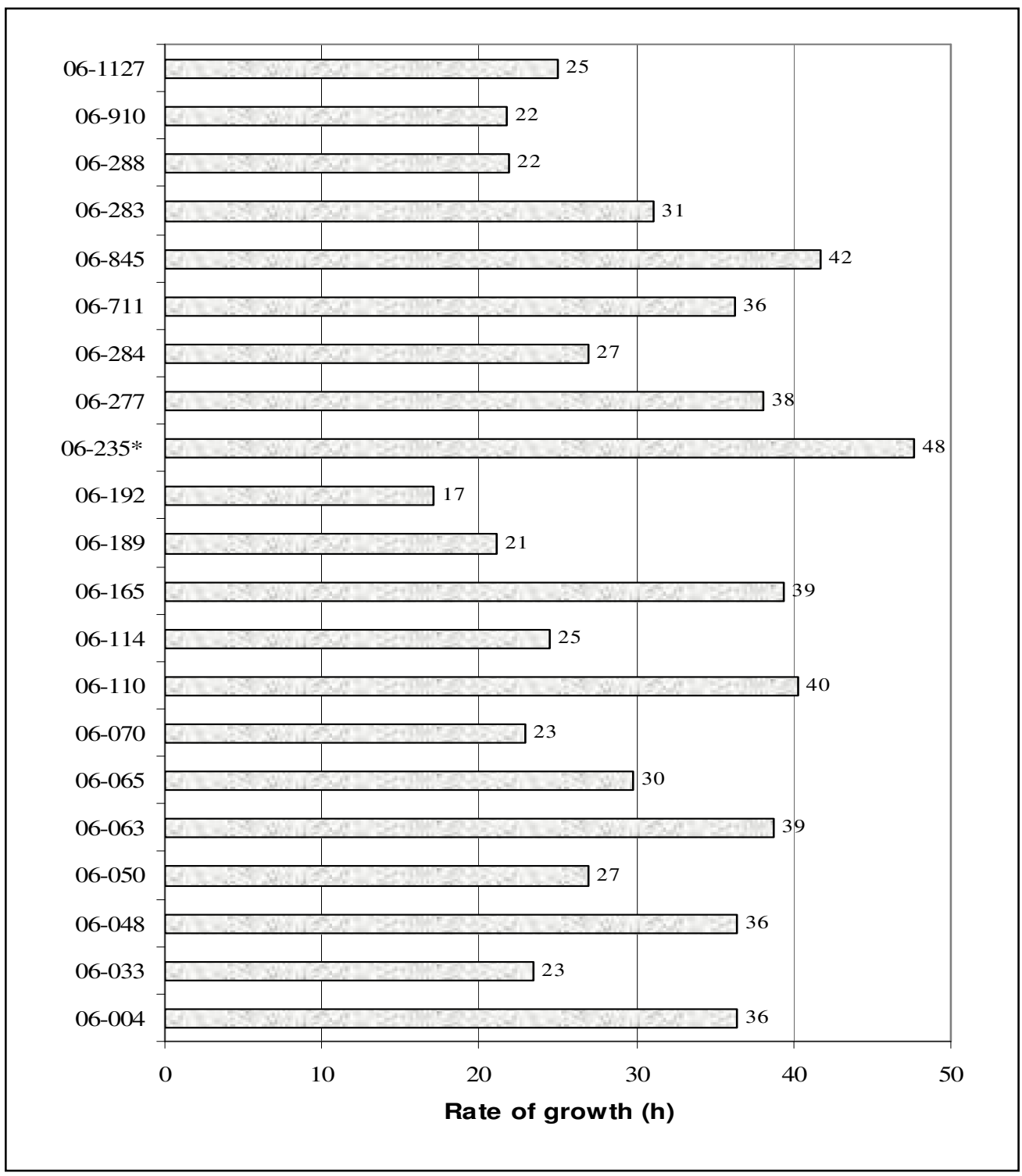

Figure 1. Rate of growth of each M. tuberculosis clinical isolates. * Strain MDR 06-235 presented the slowest rate of growth among the 21 clinical isolates. 
In summary we have standardized a simple, rapid and inexpensive method for the measurement of mycobacterial growth and its metabolism. This method showed its usefulness in assessing the fitness of mycobacteria and could be helpful in studies to understand the spreading capacity of M. tuberculosis isolate in a determined population.

\section{ACKNOWLEDGEMENTS}

This study was partially supported by the Brazilian Health Ministry and CNPq grant MCT- CNPq / MS-SCTIE-DECIT $\mathrm{N}^{\mathrm{o}}$ 25/2006 and EC project HEALTH-F3-2007-201690 (FAST-

\section{XDR-DETECT).}

A. Von Groll was partially supported by UNESCOL'Oréal Co-Sponsored Fellowship for Young Women in Life Science and Belgium Federal Government Grant Programmatorische Federale Overheidsdienst Wetenschapsbeleid contract No. BL/09/BR2.

\section{REFERENCES}

1. Ali-Vehmas, T.; Louhi, M.; Sandholm, M. (1991). Automation of the resazurin reduction test using Fluormetry of microtitration trays. $J$. Vet.Med. 38, 358-372.

2. Chien, H.P.; Yu, M. C.; Wu, M. H.; Lin,T. P.; Luh, K. T. (2000). Comparison of the BACTEC MGIT 960 with Löwenstein-Jensen medium for recovery of mycobacteria from clinical specimens. Int. J.Tuberc.Lung Dis. 4, 866-870.

3. Damato, J.J.; Collins, M.T.; Rothlauf, M.V.; McClatchy, J.K. (1983). Detection of mycobacteria by radiometric and standaard plate procedures. J.Clin.Microbiol. 17, 1066-1073.

4. Lambrecht, R.S.; Carriere, J.F.; Collins, M.T. (1988) A model for analysing growth kinetics of a Slowly growing mycobacterium sp. Appl.Environ.Microbiol. 54, 910-916.

5. Li, Z.; Kelley, C.; Collins, F.; Rouse, D.; Morris, S. (1996). Expression of katG in Mycobacterium tuberculosis Is Associated with Its Growth and Persistence in Mice and Guinea Pigs. J. Infect. Dis. 84, 1030-1035.

6. Martin, A.; Camacho, M.; Portaels, F.; Palomino, J.C. (2003). Resazurin microtiter assay plate testing of Mycobacterium tuberculosis susceptibilities to Secon-line Drugs: Rapid, simple and inexpensive method. Antimicrob. Agents Chemother. 47, 3616-3619.

7. Meyers, P.R.; Bourn, W.R.; Steyn, L.M.; van Helden, P.D.; Beyers,
A.D.; Brown, G.D. (1998). Novel Method for Rapid Measurement of Growth of Mycobacteria in Detergent-Free Media. J. Clin. Microbiol. 36, 2752-2754.

8. Morgan, M.A.; Horstmeier, C.D.; DeYoung, D.R.; Roberts, G.D. (1983). Comparison of a radiometric method (BACTEC) and conventional culture media for recovery of mycobacteria from smear negative specimens. J. Clin. Microbiol. 18, 689-696.

9. O’Brien, J.; Wilson, I.; Orton, T.; Pognan, F. (2000). Investigation of the Alamar Blue (resazurin) fluorescent dye for the assessment of mammalian cell cytotoxicity. Eur. J. Biochem. 267, 5421-5426.

10. Palomino, J.C.; Martin, A.; Camacho, M.; Guerra, H.; Swings, J.; Portaels, F. (2002). Resazurin microtiter assay plate: simple and inexpensive method for detection of drug resistance in Mycobacterium tuberculosis. Antimicrob. Agents Chemother. 46, 2720-2722.

11. Roberts, G.D.; Goodman, N.L.; Heifets, L.; Larsh, H.W.; Lindner, T.H.; McClatchy, J. K.; McGinnis, M.R.; Siddiqi, S.H.; Wright., P. (1983). Evaluation of the BACTEC radiometric method for recovery of mycobacteria and drug susceptibility testing of Mycobacterium tuberculosis from acid-fast smear positive specimens. J. Clin. Microbiol. 18, 689-696.

12. Sanchotene, K.O.; Von Groll, A.; Ramos, D.F.; Scholante, A.B.; Honscha, G.; Valença, M.; Scaini, C.J.; Silva, P.E.A. (2008) Comparative evaluation of the nitrate reductase assay and the resazurin microtitre assay for drug susceptibility testing of Mycobacterium tuberculosis against first line anti-tuberculosis drugs. Braz. J. Microbiol. 39, 16-20.

13. Taneja, N.K. and Tyagi, J.S. (2007). Resazurin reduction assays for screening of anti-tubercular compounds against dormant and actively growing Mycobacterium tuberculosis, Mycobacterium bovis BCG and Mycobacterium smegmatis. J. Antimicrob. Chemother. 60, 288-293.

14. Toungoussova, O.S.; Caugan, D.A.; Sandven, P.; Mariandyshev, A.O.; Bjune, G. (2004). Impact of drug resistance on fitness of Mycobacterium tuberculosis strains of the W-Beijing genotype. FEMS Immun. Med. Microb. 42, 281-290.

15. Verma, A.; Sampla, A.; Tyagi, J.S. (1999). Mycobacterium tuberculosis rrn Promoters: Differential Usage and Growth Rate-dependent control. J. Bacteriology. 181, 4326-4333.

16. Voskuil, M.I.; Visconti, K.C.; Schoolnik G.K. (2004). Mycobacterium tuberculosis gene expression during adaptation to stationary phase and low-oxygen dormancy. Tuberculosis. 84, 218-227.

17. Walters, S. B. and Hanna, B. A. (1996). Testing of susceptibility of Mycobacterium tuberculosis to isoniazid and rifampin by mycobacterium growth indicator tube method. J. Clin. Microbiol. 34, 1565-1567.

18. Zrimsek, P.; Kunc, J.; Kosec, M.; Mrkun, J. (2004). Spectrophotometric application of resazurin reduction assay to evaluate boar semen quality. Int. J. Androl. 27, 57-62. 